\title{
Uso do Doppler Transcraniano na Fase Aguda do Acidente Vascular Cerebral Isquêmico
}

\author{
The Utility of Transcranial Doppler in the Acute Ischemic Stroke
}

Viviane Flumignan Zétola e Marcos Christiano Lange

Setor de Doenças Cerebrovasculares, Serviço de Neurologia do Departamento de Clínica Médica do Hospital de Clinícas da UFPR - Curitiba, PR

Os primeiros estudos clínicos com o uso do Doppler transcraniano (DTC) durante a fase aguda do acidente vascular cerebral isquêmico (AVCi) foram realizados ainda no período pré-trombólise. Velocidade média de fluxo (VM) na artéria cerebral média (ACM) menor que $20 \mathrm{~cm} / \mathrm{seg}$ ou $50 \%$ abaixo da ACM contralateral, nas primeiras 24 horas, traduz fator de pior prognóstico ${ }^{1}$. A trombólise endovenosa no tratamento do AVCi possibilitou uma utilização ainda maior do DTC na fase aguda, complementando a avaliação emergencial, possibilitando a identificação da oclusão intracraniana e configurando-se em ferramenta única na monitoração contínua da abertura do vaso - recanalização ${ }^{2}$. Desenhos de estudos mais recentes propõem que as alterações encontradas no DTC façam parte dos critérios de inclusão no uso do trombolítico endovenoso, uma vez que esse esquema terapêutico é potencialmente indicado e benéfico nos casos de isquemia encefálica com oclusão de ramo arterial intracraniano ${ }^{3-5}$.

O DTC vem sendo absorvido pelos principais serviços universitários do nosso país e também por hospitais que realizam atendimento de pacientes com doenças cerebrovasculares ${ }^{6}$. Além da não-invasibilidade, o DTC apresenta como principais vantagens o baixo custo, o dinamismo, a rapidez em casos direcionados e a portabilidade. Pode ser realizado e repetido inúmeras vezes, à beira de leito, podendo ainda apresentar potencial terapêutico ${ }^{7-9}$. As principais dificuldades do método são a de pertencer ao grupo de exames complementares operador-dependente e a falta de estudos para estabelecimento de critérios de interpretação uniformemente aceitos ${ }^{7}$. Na situação do AVCi agudo, em paralelo aos exames de imagem, como a tomografia axial computadorizada (TAC) e a ressonância nuclear magnética (RNM), o DTC vem inovando conceitos e tratamentos permitindo melhor atendimento aos pacientes candidatos e não-candidatos à trombólise. A pesquisa de microembolias - ferramenta única - e a alta sensibilidade para detecção e seguimento de estenoses intracranianas fazem do DTC um exame de rotina no paciente com doença cerebrovascular $(\mathrm{DCV})^{3,10}$. Limitaremos essa revisão em situação de atendimento agudo da DCV.

É importante ressaltarmos que os principais estudos estão relacionados com a análise da ACM, cuja topografia corresponde à maioria dos eventos isquêmicos cerebrais. Sugerimos, sempre que possível, a investigação extracraniana não-invasiva com ultra-sonografia dos vasos cervicais - artérias carótidas, vertebrais e subclávias - para o auxílio na interpretação e conseqüente aumento da acurácia do DTC ${ }^{11}$.

\section{Trombólise}

Embora ainda sejam poucos os serviços que disponibilizam tratamento trombolítico aos pacientes com AVCi agudo em nosso país, a tendência em aprimorar essa terapêutica é imperativa e promissora. Estudos post-hoc deixam claro que, apesar do melhor prognóstico na maioria dos pacientes submetidos à trombólise, quando comparados ao placebo, um grupo significativo permanece sem apresentar benefícios, demonstrando um efeito terapêutico seletivo baseado em determinadas características do AVCi agudo ${ }^{12,13}$. Ao nos reportarmos aos estudos pioneiros coronarianos, observamos que o principal fator relacionado à falha da trombólise é a característica da circulação próxima ao vaso trombosado, a qual é graduada através do fluxo presente na arteriografia, denominada TIMI (thrombolysis in myocardial ischemia) ${ }^{14}$.

Utilizando essa classificação como modelo, Demchuk e cols. ${ }^{4}$, em 2001, descreveram uma análise sistemática da ACM por meio do DTC no AVCi agudo denominada TIBI (thrombolysis in brain ischemia) (Tab. 1) 3 $^{3}$ O objetivo é a determinação da velocidade de fluxo residual e sua correlação com a gravidade do AVCi, incluindo a independência funcional após o evento isquêmico inicial. A principais limitações são a ausência de fluxo no vaso - "grau 0", sendo necessário diferenciá-lo da dificuldade de transmissão do ultra-som através da porção escamosa do osso temporal, presente em aproximadamente $11 \%$ dos pacientes, e a inexistência de padrão de referência para comparação do método ${ }^{4,6}$. Com equipe treinada e utilizando-se protocolo padronizado, o DTC pode ser realizado em tempo inferior a 15 minutos, não interferindo na janela terapêutica para trombólise ${ }^{15}$.

Vários estudos experimentais já demonstraram que o ultra-som facilita a atividade de agentes fibrinolíticos em razão da melhora no transporte da medicação, alteração da estrutura da fibrina e aumento da ligação tPA-fibrina ${ }^{8}$. O estudo

\section{Palavras-chave \\ Ultra-sonografia, ultra-sonografia Doppler transcraniana, acidente cerebrovascular.}




\section{Artigo de Revisão}

\begin{tabular}{|c|c|c|}
\hline Grau & Padrão da VF & Características \\
\hline 0 & Ausente & 1) Ausência de sinal de VF apesar de diferentes graus de background noise. \\
\hline 1 & Mínimo & $\begin{array}{l}\text { 1) Pico sistólico de velocidade e duração variáveis. } \\
\text { 2) Ausência de VF diastólica durante todo o ciclo cardíaco baseado na interpretação } \\
\text { visual de períodos de ausência de fluxo durante o final da diástole. } \\
\text { (fluxo reverberante é um padrão mínimo) }\end{array}$ \\
\hline 2 & $\begin{array}{l}\text { Achatado } \\
\text { (do inglês: blunted) }\end{array}$ & $\begin{array}{l}\text { 1) Aceleração sistólica atrasada ou achatada de duração variável comparada ao controle. } \\
\text { 2) Velocidade diastólica final positiva. } \\
\text { 3) IP }<1,2 \text {. }\end{array}$ \\
\hline 3 & $\begin{array}{l}\text { Reduzido } \\
\text { (do inglês: dampened) }\end{array}$ & $\begin{array}{l}\text { 1) Aceleração sistólica normal. } \\
\text { 2) Velocidade diastólica final positiva. } \\
\text { 3) Redução da velocidade média de fluxo } \geq 30 \% \text { comparada ao vaso contralateral } \\
\text { (controle). }\end{array}$ \\
\hline 4 & Estenose & $\begin{array}{l}\text { 1) Velocidade média de fluxo }>80 \mathrm{~cm} / \mathrm{seg} \text { e diferença }>30 \% \text { comparada ao controle. } \\
\text { 2) Se a diferença entre as velocidades é menor que } 30 \% \text {, observar sinais de } \\
\text { turbulência. } \\
\text { 3) Se a velocidade em ambos os lados é menor que } 80 \mathrm{~cm} / \mathrm{seg} \text {, observar diferença de } \\
\text { 30\% e sinais de turbulência. }\end{array}$ \\
\hline 5 & Normal & $\begin{array}{l}\text { 1) Diferença com lado controle }<30 \% \text {. } \\
\text { 2) Espectro de onda similar em ambos os lados. }\end{array}$ \\
\hline \multicolumn{3}{|c|}{$V F=$ Velocidade de fluxo; $I P=$ Índice de pulsatilidade. } \\
\hline
\end{tabular}

CLOTBUST demonstrou uma possível potencialização da trombólise quando utilizada concomitantemente com o DTC, nos casos de documentação de oclusão de ACM com menos de três horas de evolução. O grupo monitorado continuamente durante a infusão endovenosa do trombolítico apresentou um maior índice de recanalização e melhora da independência funcional, quando comparado aos pacientes que não foram monitorados continuamente com o DTC ${ }^{5}$. Entretanto, estudo recente, denominado TRUMBI, onde os autores analisaram a relação da baixa intensidade do ultra-som (300 Khz) com o potencial de trombólise, foi interrompido precocemente pela maior incidência de hemorragia intraparenquimatosa sintomática no grupo de estudo ${ }^{16}$. Outros estudos serão necessários para a fundamentação desse método. A utilização de solução de microbolhas endovenosa tem demonstrado aumentar ainda mais o efeito obtido pela associação do DTC com o trombolítico ${ }^{17}$.

Entre as informações obtidas pelo método, o DTC também possibilita o reconhecimento da recanalização precoce na ACM ocluída após a infusão do trombolítico, independente da recuperação clínica, conhecido como cérebro hibernado ${ }^{15,18}$. O padrão de recanalização, quando espontânea, deve sempre ser diferenciado de estenose intracraniana, a qual pode apresentar-se com achados similares no exame inicial. Os exames seqüenciais serão fundamentais para definição, uma vez que na recanalização as velocidades de fluxo retornam a valores próximos à normalidade em um período variável de tempo.

\section{Monitoração de microembolias}

O DTC possibilita a identificação de sinais de microembolia, do inglês "microembolic signs" (MES), na circulação intracraniana. Esse estudo é possível, pois a ultra- sonografia diferencia as características do sinal emitido por materiais embólicos - sólidos ou gasosos - da velocidade de fluxo das hemáceas, sendo essas diferenças presentes no espectro de onda - intensidade e freqüência - e na sonoridade emitida ${ }^{19}$.

A embolia pode originar-se de fontes cardíacas, das grandes artérias - aorta, carótidas e vertebrais -, artérias intracranianas, e paradoxalmente alcança a circulação cerebral por meio da presença de shunt venoso-arterial - forame oval patente ou fístula arteriovenosa ${ }^{20}$. A fonte cardioembólica representa $20 \%$ a $30 \%$ dos AVCi, sendo a maioria dos pacientes identificados presuntivamente conforme os critérios de TOAST, pela combinação da sintomatologia clínica associada aos fatores de médio e alto risco provenientes dos achados de exames complementares cardiovasculares - ECG, ecocardiograma transtorácico ou transesofágico - e exames de imagem - TAC e RNM - de crânio ${ }^{21}$. Nenhum desses métodos, no entanto, possibilita a identificação direta do fenômeno embólico em atividade como demonstrado objetivamente por meio do DTC.

A metodologia inclui a monitoração simultânea de ambas as ACM por um período mínimo de 30 minutos, estando os transdutores fixados para diminuir artefatos de movimento. $\mathrm{Na}$ presença de duas possíveis fontes embólicas, cardiogênica e placa carotídea, a identificação de MES permite um aumento da acurácia diagnóstica e um auxílio na decisão terapêutica, além de sua detecção - MES - ser um fator preditor na recorrência de um novo evento isquêmico cerebral ${ }^{20,22-24}$.

Linhas de pesquisa com a monitoração de procedimentos cirúrgicos e endovasculares vêm sendo estabelecidas, correlacionando a presença de MES e alterações neurológicas no pós-operatório ${ }^{25-29}$. Esse procedimento poderá, futuramente, 
reconhecer pontos cruciais na manipulação cirúrgica das estenoses carotídeas.

O avanço técnico do DTC propiciará melhor conhecimento da fisiopatologia do fenômeno embólico, e provavelmente será de grande auxílio guiando a terapêutica mais adequada. Nesse momento, podemos considerar que o estudo para pesquisa de microembolia é útil em pacientes com AVCi indefinido de provável etiologia cardioembólica e em portadores de aterosclerose de grandes vasos ${ }^{30,31}$.

\section{Estenoses intracranianas}

A doença intracraniana corresponde a aproximadamente $8 \%$ dos AVCi, dependendo do sexo e da raça ${ }^{32,33}$. O diagnóstico é freqüentemente realizado por arteriografia, exame padrão de referência, cujos riscos e invasibilidade do procedimento não permitem sua utilização em grande escala, provavelmente subestimando esses números. Avanços de técnicas diagnósticas como a angioressonância, a angiotomografia e o DTC tendem a confirmar a importância dessa etiologia como fator de risco independente para o AVC, embora ainda careçam de critérios validados na literatura ${ }^{34,35}$.

O DTC configura uma ferramenta auxiliar nesse diagnóstico apresentando sensibilidade e especificidade elevados quando são seguidos critérios rigorosos ${ }^{36}$. A sensibilidade é de $70 \%$ a $90 \%$, e a especificidade de $90 \%$ a $95 \%$ para as artérias da circulação anterior e um pouco menor para as artérias da circulação posterior; nesse território a sensibilidade de 50\% a $80 \%$, e a especificidade é de $80 \%$ a $96 \%{ }^{37}$. É importante ressaltar que, na prática, a análise da VM na identificação das estenoses intracranianas não é suficiente, sendo necessária combinação dessa com outros parâmetros, como assimetria, elevações segmentares, análise espectral e conhecimento da circulação extracraniana.

O principal fator confusional na interpretação das velocidades é a presença de circulação colateral ou de vasodilatação como mecanismos compensatórios nos casos associados com estenoses extracranianas ${ }^{38}$. O achado compatível com estenose intracraniana na fase aguda do AVCi deve ser diferenciado da "estenose evanescente". Essa terminologia define o processo de recanalização do vaso ocluído, que se comporta, hemodinamicamente, como uma estenose. Há necessidade de exames evolutivos para diferenciação diagnóstica ${ }^{10,39}$.

O DTC, quando comparado a outros métodos nãoinvasivos emergentes, traz a vantagem de também monitorar microêmbolos no sítio da estenose, o que é freqüentemente observado na nossa experiência, podendo influenciar na decisão terapêutica ${ }^{40}$.

O uso do DTC na fase aguda do AVCi propicia, portanto, uma complementação diagnóstica de valor ainda subestimado na prática, auxiliando os exames de imagem na etiologia e fisiopatologia, guiando o neurologista na conduta terapêutica e provavelmente estimando o prognóstico desses pacientes.

\section{Potencial Conflito de Interesses}

Declaro não haver conflitos de interesses pertinentes.

\section{Referências}

1. Halsey Jr JH, Tan MJ. Evaluation of acute stroke. In: Newell DW, Aaslid R (eds). Transcranial Doppler. New York: Raven Press; 1992. p. 145-51.

2. Baruzzi AC, Knobel E, Cirenza C, Kihara EN, Souza VC, Massaro A, et al. Use of tissue plasminogen activator factor for acute ischemic stroke. Arq Bras Cardiol. 1997; 68: 347-51

3. Arenillas JF, Molina CA, Montaner J, Abilleira S, González-Sánchez MA Álvarez-Sabín J. Progression and clinical recurrence of symptomatic middle cerebral artery stenosis: a long-term follow-up transcranial Doppler ultrasound study. Stroke. 2001; 32: 2898-904

4. Demchuk AM, Burgin WS, Christou I, Felberg RA, Barber PA, Hill MD, et al. Thrombolysis in brain ischemia (TIBI) transcranial Doppler flow grades predict clinical severity, early recovery, and mortality in patients treated with intravenous tissue plasminogen activator. Stroke. 2001; 32: 89-93.

5. Alexandrov AV, Molina CA, Grotta J, Garami Z, Ford SR, Alvarez-Sabin J, et al. Ultrasound-enhanced systemic thrombolysis for acute ischemic stroke. N Engl J Med. 2004; 351: 2170-8.

6. Zétola VF, Lange MC, Muzzio JA, Marchioro I, Nóvak EM, Werneck LC. Doppler transcraniano na prática neurológica. Arq Neuropsiquiatr. 2006; 64: 100-3.

7. Demchuk AM, Alexandrov AV. Acute ischemic stroke. In: Alexandrov A (ed). Cerebrovascular ultrasound in stroke prevention and treatment. New York: Blackwell Publishing/Futura; 2004. p. 170-80.

8. Francis CW. Ultrasound-enhanced thrombolysis. Echocardiography. 2001; 18: $239-46$

9. Egido JA, Sánchez C. Neurosonology in cerebral ischemia: future application of transcranial Doppler in acute stroke. Cerebrovasc Dis. 2001 11(suppl 1): 15-9.

10. Segura T, Serena J, Castellanos M, Teruel J, Vilar C, Dávalos A. Embolism in acute middle cerebral artery stenosis. Neurology. 2001; 56: 497-501.

11. Chernyshev OY, Garami Z, Calleja S, Song J, Campbell MS, Noser CA, et al. Yield and accuracy of urgent combined carotid/transcranial ultrasound testing in acute cerebral ischemia. Stroke. 2005; 36: 32-7.

12. del Zoppo GJ, Poeck K, Pessin MS, Wolpert SM, Furlan AJ, Ferbert A, et al Recombinant tissue plasminogen activator in acute thrombotic and embolic stroke. Ann Neurol. 1992; 32: 78-86.

13. Caplan LR, Mohr JP, Kistler JP, Koroshetz W. Should thrombolytic therapy be the first-line treatment for acute ischemic stroke? N Engl J Med. 1997; 337 1309-13.

14. Hackworthy RA, Sorensen SG, Fitzpatrick PG, Barry WH, Menlove RL, Rothbard RL, et al. Dependence of assessment of coronary artery reperfusion during acute myocardial infarction on angiographic criteria and interobserver variability. Am J Cardiol. 1988; 62: 538-42.

15. Alexandrov A. Ultrasound identification and lysis of clots. Stroke. 2004 35(Suppl 1): 2722-5.

16. Daffertshofer M, Gass A, Ringleb P, Sitzer M, Sliwka U, Els T, et al. Transcranial low-frequency ultrasound-mediated thrombolysis in brain ischemia: increased risk of hemorrhage with combined ultrasound and tissue plasminogen activator: results of a phase II clinical trial. Stroke. 2005; 36: 1441-6.

17. Molina CA, Ribo M, Rubiera M, et al. Microbubble administration 
accelerates clot lysis during continuous 2-MHz ultrasound monitoring in stroke patients treated with intravenous tissue plasminogen activator. Stroke. 2006; 37: 425-9.

18. Alexandrov AV, Hall CE, Labiche LA, Wojner AW, Grotta JC. Ischemic stunning of the brain. Early recanalization without immediate clinical improvement in acute ischemic stroke. Stroke. 2004; 35: 449-52.

19. Segura T, Serena J. Detección de microembolias mediante DTC. In: Molina CA, Serena J, Alvarez Sabín J (eds). Manual de Doppler transcraneal. Madrid: Aula Medica Ediciones; 2000. p. 51-60.

20. Markus H. Microembolic signal detection in cerebrovascular disease. In Babikian VL, Wechsler LR, Toole JF (eds). Transcanial Doppler ultrasonography. 2nd edition. Woburn: Butterworth-Heinemann; 1999. p. 167-74.

21. Adams HP Jr, Bendixen BH, Kappelle LJ, Biller J, Love BB, Gordon DL, et al. Classification of subtype of acute ischemic stroke. Definitions for use in a multicenter clinical trial. TOAST. Trial of Org 10172 in Acute Stroke Treatment. Stroke. 1993; 24: 35-41.

22. Batista P, Oliveira V, Ferro JM. The detection of microembolic signals in patients at risk of recurrent cardioembolic stroke: possible therapeutic relevance. Cerebrovasc Dis. 1999; 9: 314-9.

23. Markus HS, MacKinnon A. Asymptomatic embolization detected by Doppler ultrasound predicts stroke risk in symptomatic carotid artery stenosis. Stroke. 2005; 36: 971-5.

24. Valton L, Larrue V, le Traon AP, Massabuau P, Geraud G. Microembolic signals and risk of early recurrence in patients with stroke or transient ischemic attack. Stroke. 1998; 29: 2125-8.

25. Spencer MP, Thomas GI, Nicholls SC, Sauvage LR. Detection of middle cerebral artery emboli during carotid endarterectomy using transcranial Doppler ultrasonography. Stroke. 1990; 21: 415-23.

26. Siebler M, Sitzer M, Rose G, Bendfeldt D, Steinmetz H. Silent cerebral embolism caused by neurologically symptomatic high-grade carotid stenosis. Event rates before and after carotid endarterectomy. Brain. 1993; 116 (Pt 5): 1005-15.

27. Markus HS, Thomson N, Brown MM, Thomson ND. Asymptomatic cerebral embolic signals in symptomatic and asymptomatic carotid artery disease. Brain. 1995; 118: 1005-11.

28. Siebler M, Nachtmann A, Sitzer M, Rose G, Kleinschmidt A, Rademacher J, et al. Cerebral microembolism and the risk of ischaemia in asymptomatic high-grade internal carotid artery ischaemia. Stroke. 1995; 26: 2184-6.
29. Malheiros SM, Massaro AR, Gabbai AA, Pessa CJ, Gerola LR, Branco JN, et al. Is the number of microembolic signals related to neurologic outcome in coronary bypass surgery? Arq Neuropsiquiatr. 2001; 59: 1-5.

30. Alexandrov AV, Neumyer MM. Diagnostic criteria for cerebrovascular ultrasound. In: Alexandrov A, ed. Cerebrovascular ultrasound in stroke prevention and treatment. New York: Blackwell Publishing/Futura; 2004. p. 107-8.

31. Ringelstein EB, Droste DW, Babikian VL, Evans DH Grosset DG, Kaps M, et al. Consensus on microembolus detection by TCD. International Consensus Group on Microembolus Detection. Stroke. 1998; 29: 725-9.

32. Sacco RL, Kargman DE, Gu Q, Zamanillo MC. Rate-ethnicity and determinants of intracranial atherosclerotic cerebral infarction. Stroke. 1995; 26: 14-20.

33. Wityk RJ, Lehman D, Klag M, Coresh J, Ahn H, Litt B. Race and sex differences in the distribution of cerebral atherosclerosis. Stroke. 1996; 27: 1974-80.

34. Wong KS, Li H, Chan YL, Ahuja A, Lam WWM, Wong A, et al. Use of transcranial Doppler ultrasound to predict outcome in patients with intracranial large-artery occlusive disease. Stroke. 2000; 31: 2641-7.

35. Chimowitz MI, Kokkinos J, Strong J, Brown MB, Levine SR, Silliman S, et al. The Warfarin-Aspirin symptomatic in,tracranial disease study. Neurology. 1995; 45: 1488-93.

36. Felberg RA, Christou I, Demchuk AM, Malkoff M, Alexandrov AV. Screening for intracranial stenosis with transcranial Doppler: the accuracy of mean flow velocity tresholds. J Neuroimaging. 2002; 12: 1-6.

37. Sloan MA, Alexandrov AV, Tegeler CH, Spencer MP, Caplan LR, Feldman $\mathrm{E}$, et al. Assessment transcranial Doppler ultrasonography report of the Therapeutics and Technology Assessment Subcommittee of the American Academy of Neurology. Neurology. 2004; 62: 1468-81.

38. Rorick MB, Nichols FT, Adams RJ. Transcranial Doppler correlation with angiography in detection of intracranial stenosis. Stroke. 1994; 25: 1931-4.

39. Diehl RR, Samii C, Diehl A. Dynamics and embolic activity of symptomatic intra-cranial cerebral artery stenoses. Acta Neurol Scand. 2002; 106: 173-81.

40. Gao S, Wong KS, Hansberg T, Lam WW, Droste DW, Ringelstein EB. Microembolic signal predicts recurrent cerebral ischemic events in acute stroke patients with middle cerebral artery stenosis. Stroke. 2004; 35 : 2832-6. 